\title{
Positron Annihilation Method: Thermal and Non-Thermal Defects in Refractory Intermetallics
}

\author{
Y. Shirai \\ Department of Materials Science and Engineering, \\ Osaka University, Suita Osaka 565, Japan
}

(Received September 17, 1997)

\begin{abstract}
Positron applications to study defects in high temperature materials are reviewed. Special emphasis is put on some refractory intermetallic compounds. After an introduction to positron annihilation methods which have been successfully applied to the studies of atomic-scale defects in solids, in-situ measurements of thermal equilibrium defects in some intermetallics are reviewed. Studies of constitutional defects and non-equilibrium defects in intermetallic compounds are also presented. The last part of this article introduces a new positron apparatus which will become a very powerful tool in studying thermal defects at high temperatures in refractory materials.
\end{abstract}

\section{INTRODUCTION}

The positron is the antiparticle to the electron. The positron has the same rest mass $\left(m_{0}\right)$ as the electron but the opposite charge; that is, one positive elementary charge. In condensed matter, each positron annihilates with an electron into gamma-rays in a very short time $\left(10^{-10}-10^{-9} \mathrm{~s}\right)$. By measuring the angular correlation or the Doppler broadening of the emitted gamma-quanta, or by measuring the positron lifetime, we can deduce the information about electron momentum distribution or electron density at the positron annihilation sites, which are interstitial sites of crystals, crystal lattice defects, or free surfaces of the materials.
Positron applications to the study of crystal lattice defects started around 1970, when it was recognized correctly that positrons are sensitively trapped by vacancy-type defects and annihilate with characteristics of each type of defects. Since then, there has been a growing interest in positron spectroscopy of defects in solids and now the number of publications in the field is much higher than that of the original fields of positron applications, such as the study of Fermi surfaces in metals and alloys. This fact clearly demonstrates the unique usefulness of positrons in studies of lattice defects.

In this short review, we focus our attention on the field of positron application to probe into defects in intermetallic compounds, which have received considerable interest in recent years as high-temperature structural materials. The readers are referred to some textbooks $/ 1-3 /$, and to recent proceedings $/ 4-6 /$ for general information on the whole region of positron solid-state physics.

\section{POSITRON ANNIHILATION}

There are many radio isotopes that decay with the emission of positrons. In conventional positron annihilation experiments, positrons, which can be readily obtained from such radioactive isotopes, are directly injected into a specimen. The most popular positron sources are ${ }^{22} \mathrm{Na},{ }^{58} \mathrm{Co},{ }^{64} \mathrm{Co}$ and ${ }^{68} \mathrm{Ge}$. The implantation depth of positrons is in the range of 
100-1000 $\mu \mathrm{m}$, depending on the positron energy and the density of the sample. Hence, positrons deduce information in the bulk of a specimen in conventional source experiments. Surface or near-surface information is obtained by use of recent slow-positron beam techniques /7-9/.

Positrons injected in materials lose their kinetic energy $\left(10^{5}-10^{6} \mathrm{eV}\right.$ depending on source nuclei) and slow down to the thermal energy $(k T)$ (thermalization) within a few picoseconds and diffuse in the specimen. Positron lifetimes in well-annealed metallic materials are in the range of $100-200 \mathrm{ps}$, as will be mentioned below. Typical diffusion length of thermalized positrons is of the order of $10^{-7} \mathrm{~m}$. In condensed matter, nearly all (about $99.7 \%$ ) positrons annihilate each with an electron into two gamma-quanta. Since the total energy and the total momentum are conserved before and after the annibilation of a positron-electron pair, the two gamma-quanta have a total energy of $2 m_{0} c^{2}=2 \times 0.511$ $\mathrm{MeV}$ ( $c$ is the velocity of light) and are emitted from the annihilation site in almost opposite directions.

The most popular positron source is ${ }^{22} \mathrm{Na}$, since the half-life of the radioisotope is as long as $\mathbf{2 . 6}$ years, and the efficiency of the $\beta^{+}$decay (positron emission) is as high as $90 \%$. Moreover, ${ }^{22} \mathrm{Na}$ is convenient for positron lifetime measurements, because it emits a high-energy $\gamma$-ray $(1.28 \mathrm{MeV})$ immediately after the emission of a positron (within a few picosecond). This $\gamma$-ray of 1.28 $\mathrm{MeV}$ is practically used as the signal of a positron emission. Positron lifetime is obtained by measuring the time difference between the detection of the $1.28 \mathrm{MeV}$ g-ray and the detection of the $0.511 \mathrm{MeV} \gamma$-ray, which is emitted by the positron-electron pair annihilation. The annihilation rate $(\lambda)$ is proportional to the electron density at the annihilation site. Positron lifetimes $\tau$ $\left(=\lambda^{-1}\right)$ in well-annealed metallic materials are characteristic of each material and in the range of 1-2 $x$ $10^{-10}$ sec.

The momentum $p$ of a positron-electron pair is also conserved after the annihilation. Therefore, two annihilation gamma-quanta are emitted in opposite directions with an angular deviation from $\pi$ by $p_{1} / m_{0} c$, where $p_{1}$ is the perpendicular component of $p$. Since the momentum of a thermalized positron is negligibly small compared with that of an electron in. a crystal, the angular deviation gives us the momentum of the electron in the specimen. Thus, we can obtain the electron momentum distribution in a sample by measuring the angular distribution of coincidence-count rates of annihilation gamma-rays.

On the other hand, the parallel component of the momentum $p_{2}$ of an electron-positron pair to the main direction of annihilation photons gives rise to the Doppler broadening $\left(p_{2} c / 2\right)$ of the energy $(0.511 \mathrm{MeV})$ of the annihilation photons. Thus, it is also possible to know the electron momentum distribution in a sample by precise measurements of the Doppler broadening of annibilation photons. The resolution, however, is more than one order of magnitude lower than in angular correlation measurements, owing to the limitation of energy resolution of available detector systems. On the contrary, data accumulation rates by the Doppler broadening method are usually much higher than by angular correlation methods.

In a perfect crystal, thermalized positron is delocalized (in a Bloch function state). The density has maxima in the interstices, since a positron is strongly repelled by ion cores due to the Coulomb repulsion. When there exist vacancies or vacancy clusters or other defects which have lower densities of ion cores than the surroundings, positrons are trapped in the defects and annihilate there. In such defects, positron lifetime is longer, because electron density (both conduction and core electrons) is lower. On the hand, the angular correlation curve and the Doppler broadening spectrum become narrower and the peak counting rate becomes higher. These positron annihilation characteristics are unique for each kind of defects, and thus make us possible to identify each kind of defects.

The positron sensitivity to such defects is quite high: In the case of vacancies, the concentration of $10^{-6}$ in atomic fraction is enough to be detected; $10^{-4}$ vacancies cause observed characteristics to be saturated with those of vacancies. The sensitivity for vacancy clusters or secondary defects is even higher. This fact is especially noteworthy, since the relative intensity of the signal of defects is equal to the defect concentration in most experimental methods other than positron annihilation. It can be said that positrons are capable of amplifying the signal of defects by the order of $10^{4}$.

For positrons annihilating in a perfect lattice, the decay is simple exponential; the positron lifetime spectrum has the form 


$$
T(t)=\exp \left(-t / \tau_{1}\right)
$$

where the $\tau_{1}$ is the positron lifetime in the perfect lattice. When positron-trapping centers such as vacancies exist in the lattice, some positrons are trapped and annihilate in them. According to the so-called trapping model $/ 10-12 /$, the positron lifetime spectrum well approximates to two discrete components:

$$
T(t)=I_{0} / \tau_{0} \exp \left(-t / \tau_{0}\right)+I_{\mathrm{d}} / \tau_{\mathrm{d}} \exp \left(-t / \tau_{\mathrm{d}}\right)
$$

where the $\tau_{d}$ is the positron lifetime in the defect which is characteristic of each kind of defects. The relative intensity of the defect

$$
I_{\mathrm{d}}=\kappa /\left(\tau_{1}^{-1}+\kappa-\tau_{\mathrm{d}}{ }^{-1}\right),
$$

where the $\kappa$ is the positron trapping rate and is proportional to the concentration of the trapping centers. The specific trapping rate $v$, that is the trapping rate per unit concentration of the defect, is dependent on the defect. Values of $v$ for monovacancies in most metals are $10^{14}-10^{15}$ per second. The decay constant $\tau_{0}$ of the other exponential term is given by $\left(\tau_{1}^{-1}+\kappa\right)^{-1}$ which is shorter than $\tau_{1}$. The mean lifetime is given by

$$
\tau_{M}=I_{0} \tau_{0}+I_{\mathrm{d}} \tau_{\mathrm{d}}
$$

The above treatment is readily extended to the cases in which more than three kinds of defects are included $/ 13,14 /$. Thus, by measuring and analyzing positron lifetime spectra, information on the concentration and nature of defects can be obtained; the defect nature via $\tau_{\mathrm{d}}$ and the concentration via $I_{\mathrm{d}}$.

On the other hand, any momentum density parameter $F$, such as the lineshape parameters $S$ or $W$ of the Doppler broadening spectrum, or the peak counting rate of the angular correlation curve $N(0)$, is a superposition of annihilations in the lattice $\left(F_{i}\right)$ and at the defects $\left(F_{\mathrm{d}}\right) / 15 /$ :

$$
F=\left(\tau_{1}^{-1} F_{1}+\kappa F_{\mathrm{d}}\right) /\left(\tau_{\mathrm{l}}^{-1}+\kappa\right) \text {. }
$$

In order to estimate the defect concentration one has to know the defect specific parameters $F_{1}$ and $F_{\text {d, }}$ which is sometimes difficult to obtain.

\section{THERMAL DEFECTS}

It is well established that almost all heat-treatments of metallic materials at high temperatures are controlled by thermal vacancies. From an experimental point of view, however, vacancies in thermal equilibrium are difficult to be measured because of the low concentration $\left(S 10^{-3}\right.$ even at the melting point of materials) and high temperature of the measurements. Only a few experimental methods can be used under thermal equilibrium conditions at high temperatures. Before the appearance of the positron annihilation method, the most successful method was the simultaneous measurement of thermal expansions of a specimen and the lattice constant as a function of temperature $/ 16 /$. The experimental precision of the method, however, is much less than positron annihilation method.

After the discovery $|10-12|$ of the reaction of positrons with vacancies in thermal equilibrium, vacancy formation enthalpies in many pure metals and alloys have been determined $/ 17 /$. Among them, most refractory metals have been studied by the Stuttgart group /18/. Positron annihilation methods have been recently applied to investigate thermal equilibrium defects in intermetallic compounds and ordered alloys. Temperature dependencies of the average positron lifetime for some intermetallics and ordered alloys are shown in Figure $1 / 19 /$. A nearly linear temperature dependence at lower temperatures is due to the thermal expansion of the crystal lattices. At higher temperatures, each plot shows an increased slope demonstrating positron trapping in thermal equilibrium vacancies. Saturation, beginning below the melting point, gives us the $S$-shaped curves as a whole. The well-developed $S$-shaped curves are clearly observed for these ordered materials.

The case of $\mathrm{Cu}_{51} \mathrm{Zn}_{49}$ serves às a good example to illustrate thermal defects in ordered materials. The crystal structure of this alloy is B2 below the order-disorder transition temperature $T c(=741 \mathrm{~K})$. The steep increase of the positron lifetime due to thermal vacancies starts at the temperature as low as $370 \mathrm{~K}$. This is a threshold temperature which measures the onset of thermal-vacancy trapping. The ratio of this threshold temperature to the melting point is only 0.32 , which is 


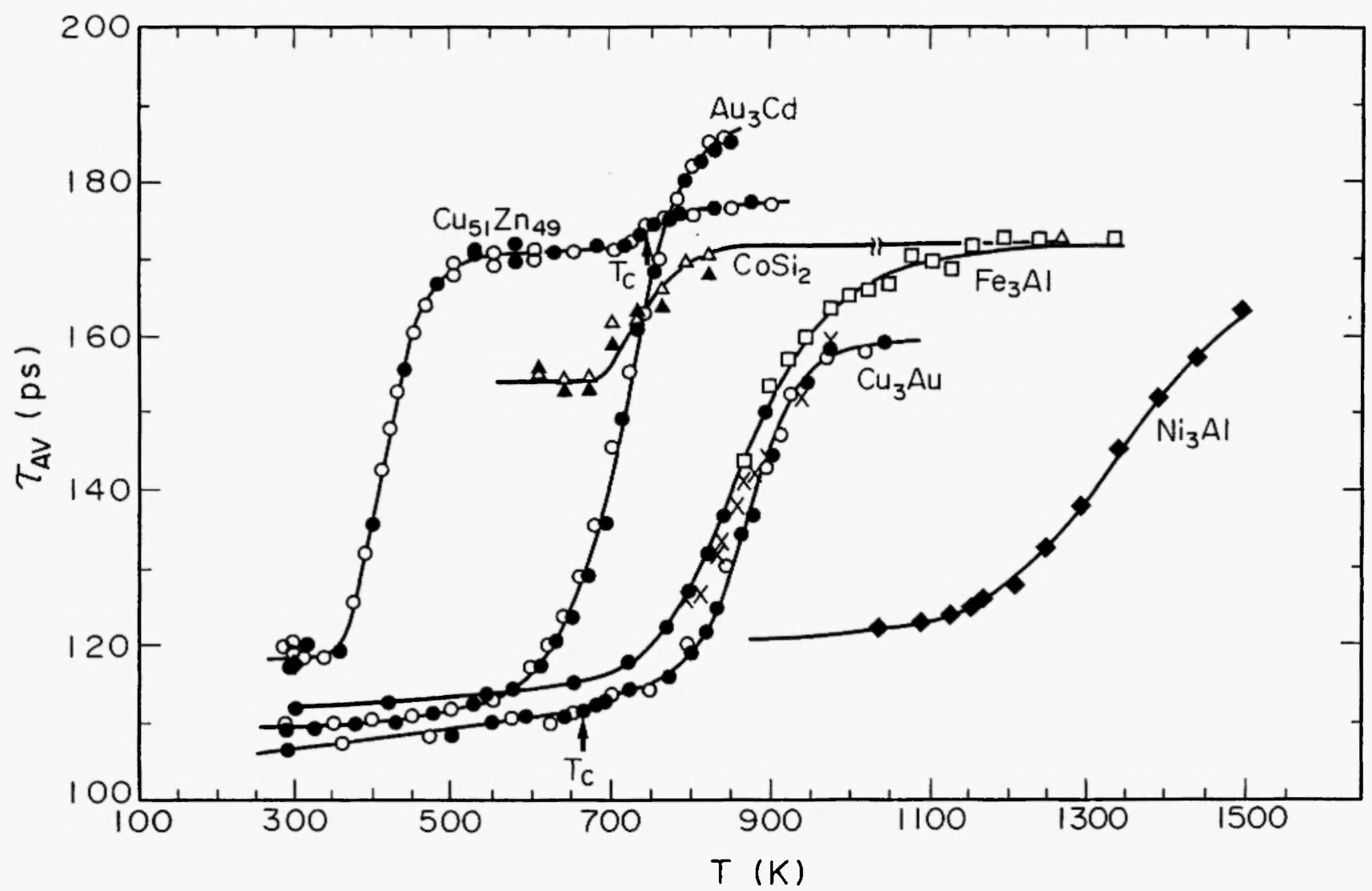

Fig.1: Measurements of thermal equilibrium vacancies with positron lifetime spectroscopy. The average positron lifetime sharply increases due to positron trapping by thermal vacancies. Open and closed circles for $\mathrm{Cu}_{51} \mathrm{Zn}_{49}$, $\mathrm{Cu}_{3} \mathrm{Au}$ and $\mathrm{Au}_{3} \mathrm{Cd}$ :heating and cooling runs, respectively; $\mathrm{Ic}$ : the order-disorder transition temperature. Different symbols for $\mathrm{Fe}_{3} \mathrm{Al}$ : different measuring runs. Open and closed triangles for $\mathrm{CoSi}_{2}$ : water quenched and air cooled specimens, respectively.

surprisingly lower than the ratio (around 0.6 ) for normal metals and disordered alloys. Around 500K and above, average positron lifetime shows saturation behavior, where almost all positrons are trapped in monovacancies and annihilate there with the characteristic lifetime (171psec). The effective vacancy formation enthalpy in ordered $\mathrm{Cu}_{51} \mathrm{Zn}_{\vartheta}$ has been determined as $0.42 \mathrm{eV}$ from the Arrhenius plot of the measured trapping rate $k$ (see Fig.2) /20/.

The effective vacancy formation enthalpies determined in the other intermetallics are $1.42 \pm 0.09 \mathrm{eV}$ in disordered $\mathrm{Cu}_{3} \mathrm{Au} / 21 /, 1.07 \pm 0.04 \mathrm{eV}$ in disordered $\mathrm{Au}_{3} \mathrm{Cd} / 22 /, 0.85 \pm 0.02 \mathrm{eV}$ in ordered $\mathrm{Au}_{3} \mathrm{Cd} / 22 /$, $1.18 \pm 0.04 \mathrm{eV}$ in $\mathrm{Fe}_{3} \mathrm{Al} / 23 /, 1.60 \pm 0.06 \mathrm{eV}$ in $\mathrm{Ni}_{3} \mathrm{Al} / 24 /$ and $1.41 \pm 0.06 \mathrm{eV}$ in TiAl $125 \%$ The last three compounds are important as high temperature materials.

It is noteworthy that the average positron lifetime in
$\mathrm{Cu}_{51} \mathrm{Zn}_{49}$ shows a significant change around the order-disorder transition temperature Tc (see Fig.1). Since this change is observed in the saturated region with vacancies, it is not due to the change in vacancy concentration but is caused by the change in positron lifetime at vacancies. Below $700 \mathrm{~K}$, most vacancies are sited on the $\mathrm{Cu}$ sublattice /26/ and trapped positrons are thus surrounded with only $\mathrm{Zn}$ atoms. As temperature approaches the order-disorder transition point, the fraction of $\mathrm{Cu}$ atoms on the $\mathrm{Zn}$ sublattice increases. Furthermore, the proportion of vacancies on the $\mathrm{Zn}$ sublattice increases $126 \%$. Above $T c$, there is no distinction between vacancies in the disordered bcc lattice and every positron trapped in a vacancy is surrounded with an equal number of $\mathrm{Cu}$ and $\mathrm{Zn}$ atoms. This change of environment surrounding positrons causes the change of the positron lifetime around 7c 


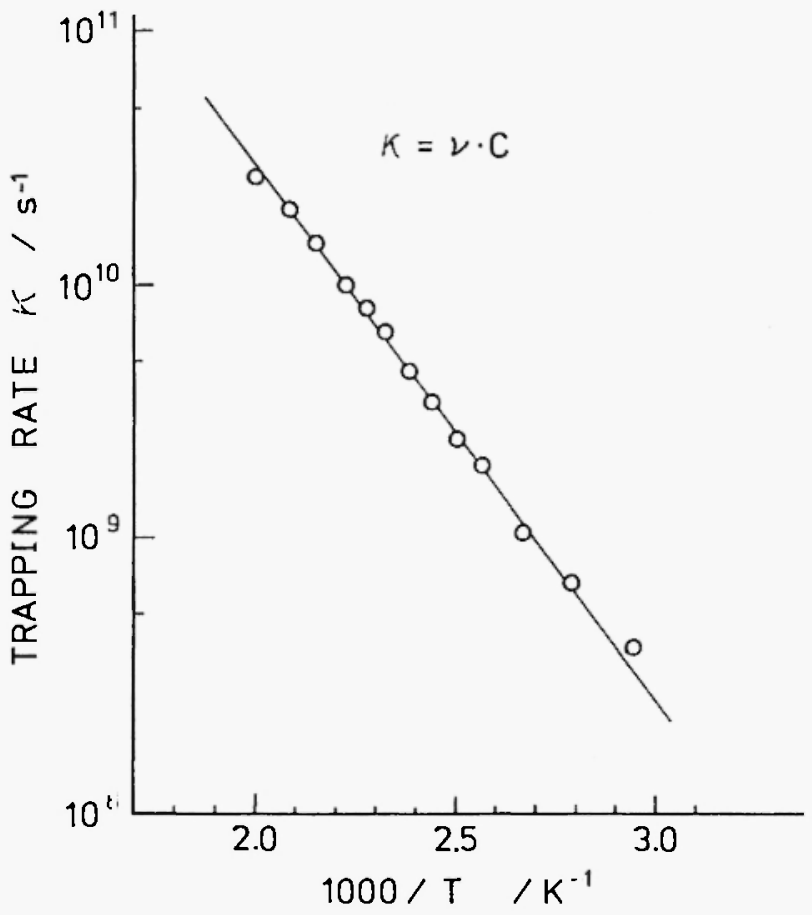

Fig.2: The Arrhenius plot of the positron trapping rate $\kappa(=v C, v$ is the specific trapping rate and $C$ is the concentration of vacancies) in $\mathrm{Cu}_{51} \mathrm{Zn}_{49}$. The effective vacancy formation enthalpy in ordered $\mathrm{Cu}_{51} \mathrm{Z}_{\mathrm{H}_{49}}$ has been determined as 0.42 $\mathrm{eV}$ by this analysis.

through the electron density change. Thus, the positron lifetime change observed around $T \mathrm{c}$ shows that positrons have the capability to distinguish vacancies on different sublattices of ordered materials.

So far, we have seen that positron lifetime spectroscopy is a sensitive and specific technique for studying thermal vacancy formation in high temperature materials. There is no principal limitation for positron annihilation methods to be used at high temperatures. It is not easy nevertheless to seal a radioactive positron source in a specimen hermetically for high temperature uses. This is the main reason why only a limited number of materials have been studied under thermal equilibrium conditions by using positron annihilation methods. A new system developed by the author's group to break through this limitation will be described in the last section of this article.

\section{CONSTITUTIONAL DEFECTS}

In intermetallic compounds or ordered alloys, different atomic species occupy differently designated lattice sites and they are usually held together by strong bonds. If an intermetallic compound has an extended phase field, its non-stoichiometry may give rise to the existence of constitutional defects such as atoms occupying atomic sites of a sublattice other than its own sublattice (antisite atoms) or vacancies of deficient atomic species (constitutional vacancies). It is well known that the type and quantity of constitutional defects directly affect mechanical properties, atomic diffusions of the host ordered materials. Which of these defects dominates the defect structure of the non-stoichiometric compound depends on the crystal structure, constituent elements, and bonding nature of the compound as well as on which of the constituent elements is deficient and the amount by which the compound is non stoichiometric /19\%.

From an experimental point of view, defect structures have been studied only in a limited number of intermetallic compounds. Among them, the existence of constitutional vacancies has been found in $\mathrm{NiAl}, \mathrm{CoAl}$, $\mathrm{CoGa}$, and $\mathrm{FeAl} / 27 /$ by measuring the lattice parameter and macroscopic length or density.

Application of the positron annihilation technique to study constitutional defects in intermetallic compounds started around 10 years ago. Shimotomai et al. 128/ measured the Doppler-broadening line shape parameter for positron annihilation in $\mathrm{NiAl}$ as a function of $\mathrm{Al}$ composition, and they confirmed the existence of a large amount of constitutional vacancies on $\mathrm{Ni}$ sublattice in the compound when $\mathrm{Ni}$ is deficient.

It is easy to distinguish which kind of constitutional defects exist in a given compound by using positron lifetime measurements. Positron lifetime values in intermetallic compounds directly reflect their defect structures. For example, all intermetallic compounds or ordered alloys shown in Fig. 1 have no constitutional vacancies. In other words, their non-stoichiometry is compensated with anti-site atoms. This is because, if constitutional vacancies exist, all positrons are trapped in such constitutional vacancies at any temperatures (concentration of constitutional vacancies is generally much higher than that of thermal vacancies) and no such 
changes as shown in Fig. 1 should be observed. In the same manner, defect structures can be easily judged by comparing positron lifetime before and after quenching or electron-irradiation, as will be shown in the next section.

Until now, $\mathrm{NiAl} / 28 /$ seems to be an almost unique example in which the existence of vacancies compensating non-stoichiometry has been proved by using a positron annihilation method. FeAl /29/ shows a somehow intermediate nature of vacancies; although there are no constitutional vacancies, the concentration of thermal vacancies is quite high and they are easily quenched-in and hardly annealed out. Similar behavior of vacancies has been also found in $\mathrm{CoSi}_{2} / 30 /, \mathrm{CO}_{2} \mathrm{Si}$ 131 / and in some other intermetallic compounds.

\section{NONEQUILIBRIUM DEFECTS}

Most heat treatments to improve properties of materials are governed by the atomic diffusion. The rate of the diffusion of substitutional atoms is determined by the migration of vacancies. The vacancy migration properties can be directly observed by the positron annihilation lifetime method.

Excess vacancies (over thermal equilibrium concentration) can be introduced in a specimen by quenching from high temperatures or by irradiation with high-energy particles such as electrons or ions. Such vacancies are well contained in the specimen at lower temperatures where vacancies are practically immobile. Above a certain temperature, vacancies become mobile by a thermal activation process and begin to diffuse. On the way to permanent sinks of vacancies such as dislocations, grain boundaries and surfaces, some vacancies encounter with each other and form less-mobile vacancy clusters or quasi-stable secondary defects, such as dislocation loops or voids. At higher annealing temperatures, the vacancy clusters or secondary defects evaporate vacancies and disappear, and the specimens recover to the thermal equilibrium state. These serial processes can be well defined by use of the positron lifetime spectroscopy, which shows us vacancy migration, vacancy clustering, formation and dissolution of secondary defects.

Fig. 3 shows mean lifetime changes as a function of annealing temperature in $\mathrm{Ti}_{44} \mathrm{Al}_{56} / 32 /$, in $\mathrm{Ti}_{51} \mathrm{Al}_{49} / 32 /$, in $\mathrm{Al}_{67} \mathrm{Mn}_{8} \mathrm{Ti}_{25} / 33 /$, in $\mathrm{CoSi}_{2} / 30 /$ and in $\mathrm{Ti}_{3} \mathrm{Al} / 34 /$, which were electron-irradiated and then isochronal annealed /19/. All the specimens were fully annealed at high temperatures and slowly cooled to room temperature before electron irradiation. In these compounds, positron lifetime is found to increase due to

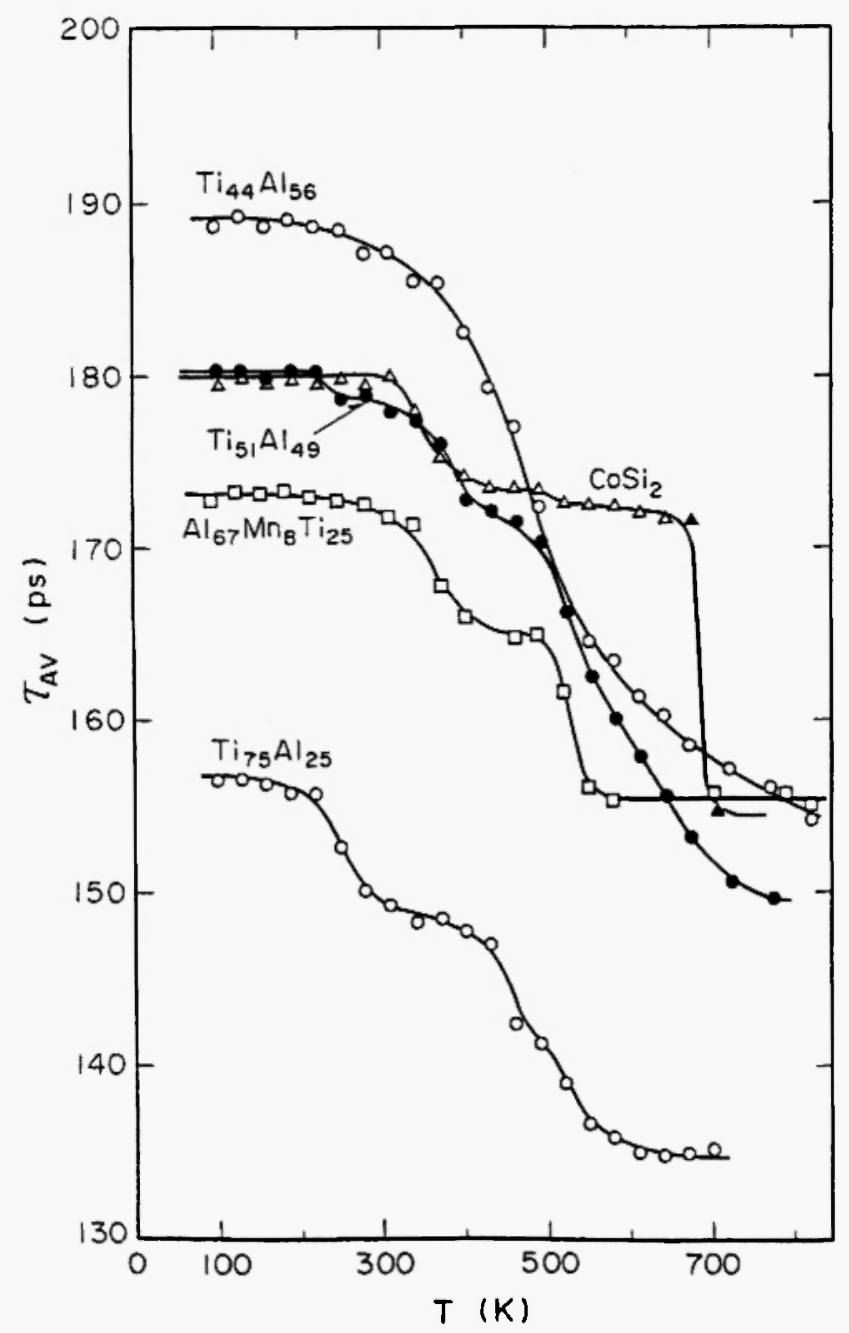

Fig.3: Vacancy migration and recovery detected with positron lifetime spectroscopy. The mean lifetime changes with isochronal annealing temperature after electron irradiations. The irradiation conditions (electron energy; total doze; irradiation temperature) are $\mathrm{Ti}_{44} \mathrm{Al}_{56}$ (10MeV; $\left.5 \times 10^{21} \mathrm{~m}^{-2}, 160 \mathrm{~K}\right), \mathrm{Ti}_{51} \mathrm{Al}_{49}$ (10MeV; $\left.5 \times 10^{21} \mathrm{~m}^{-2}, 160 \mathrm{~K}\right), \mathrm{Ti}_{3} \mathrm{Al}(1 \mathrm{MeV} ; 3$ x $\left.10^{22} \mathrm{~m}^{-2}, 150 \mathrm{~K}\right), \mathrm{Al}_{67} \mathrm{Mn}_{8} \mathrm{Ti}_{25}(1 \mathrm{MeV} ; 1.4 \mathrm{x}$ $\left.10^{22} \mathrm{~m}^{-2}, 100 \mathrm{~K}\right)$ and $\mathrm{CoSi}_{2}\left(1 \mathrm{MeV} ; 2.5 \times 10^{22}\right.$ $\left.\mathrm{m}^{-2}, 250 \mathrm{~K}\right)$. 
vacancies formed by electron irradiation. The lifetime recovers through several stages to the value in each compound at fully annealed state.

This kind of general change clearly shows that there are no constitutional vacancies in these materials shown in Fig.3. If constitutional vacancies exist, whose concentration is usually greater than $10^{-3}$, almost all positrons are trapped and annihilate at constitutional vacancies and thus practically no change in positron lifetime should be observed before and after electron irradiation, which can introduce much smaller amount of vacancies (usually about $10^{-4}$ ).

The migration and recovery stages of vacancies are clearly seen in each compound. For example, electron-irradiated $\mathrm{Ll}_{2} \quad \mathrm{Al}_{67} \mathrm{Mn}_{8} \mathrm{Ti}_{25}$ recovers in two major stages (see Fig. 3). The first stage, around $350 \mathrm{~K}$, is ascribed to the migration of vacancies. The final stage, around $500 \mathrm{~K}$, is due to the dissolution of secondary defects formed at the first stage. The secondary defects are not voids but collapsed vacancy-clusters that consist of dislocations /33/. Electron-irradiated $\mathrm{Ll}_{2} \quad \mathrm{Al}_{67} \mathrm{Mn}_{8} \mathrm{Ti}_{25}$ recovers almost to the fully annealed state below $550 \mathrm{~K}$.

More detailed information about vacancies and their clusters is obtained by the component analysis of positron annihilation spectrum. For example, lifetime spectra in electron irradiated $\mathrm{Ti}_{3} \mathrm{Al}$ specimens could be resolved into two discrete components, the longer lifetime $\left(\tau_{d}\right)$, which results from positrons trapped at defects, and the shorter lifetime $\left(\tau_{0}\right)$, which mainly comes from free positrons in the matrix (see Eq.2). The values of $\tau_{d}$ and $\tau_{0}$ are shown in Fig. 4 together with the relative intensity $I_{\mathrm{d}}$ as a function of annealing temperature. $I_{\mathrm{d}}$ is a measure of the relative number of positrons that are trapped and annihilated at defects, and hence a measure of the concentration of defects (see Eq. 3). $I_{\mathrm{d}}$ decreases as the recovery proceeds and finally disappears at about $600 \mathrm{~K}$.

Electron irradiation generated a new dominant lifetime component with the value of $185 \pm 3 \mathrm{ps}$ in $\mathrm{Ti}_{3} \mathrm{Al}$ (Fig. 4), which is distinctly higher than the value of 133 \pm 2 ps in fully-annealed $\mathrm{Ti}_{3} \mathrm{Al}$ compounds (Fig.3). This new lifetime component is ascribed to the positrons trapped and annihilated at radiation-induced $\mathrm{Ti}$ vacancies $/ 34 /$. The recovery of electron-irradiated $\mathrm{Ti}_{3} \mathrm{Al}$ starts around 200K (Fig. 3). At this stage, $\tau_{d}$ decreases but $I_{d}$ remains constant (see Fig. 4). The slight decrease in $\tau_{d}$ observed around $250 \mathrm{~K}$ (Fig. 4) is interpreted as the migration of Ti vacancies to become Al vacancies /34/.

Free migration of excess vacancies usually results in the formation of secondary defects, which survive until being annealed-out at higher temperatures by the evaporation of vacancies. It is known that a specific type of secondary defect affects positron lifetime in a specific manner: void formation increases $\tau_{d}$ and decreases $I_{\mathrm{d}}$ drastically; faulted-loop formation decreases $\tau_{d}$ by a few percent and decreases $I_{d}$ slightly, and the formation of stacking fault tetrahedra decreases $\tau_{d}$ by about $20 \%$ and decreases $I_{d}$ considerably /35/. The present observations at the stage above $350 \mathrm{~K}$ (Fig. 4) coincide well with the case of void formation. The
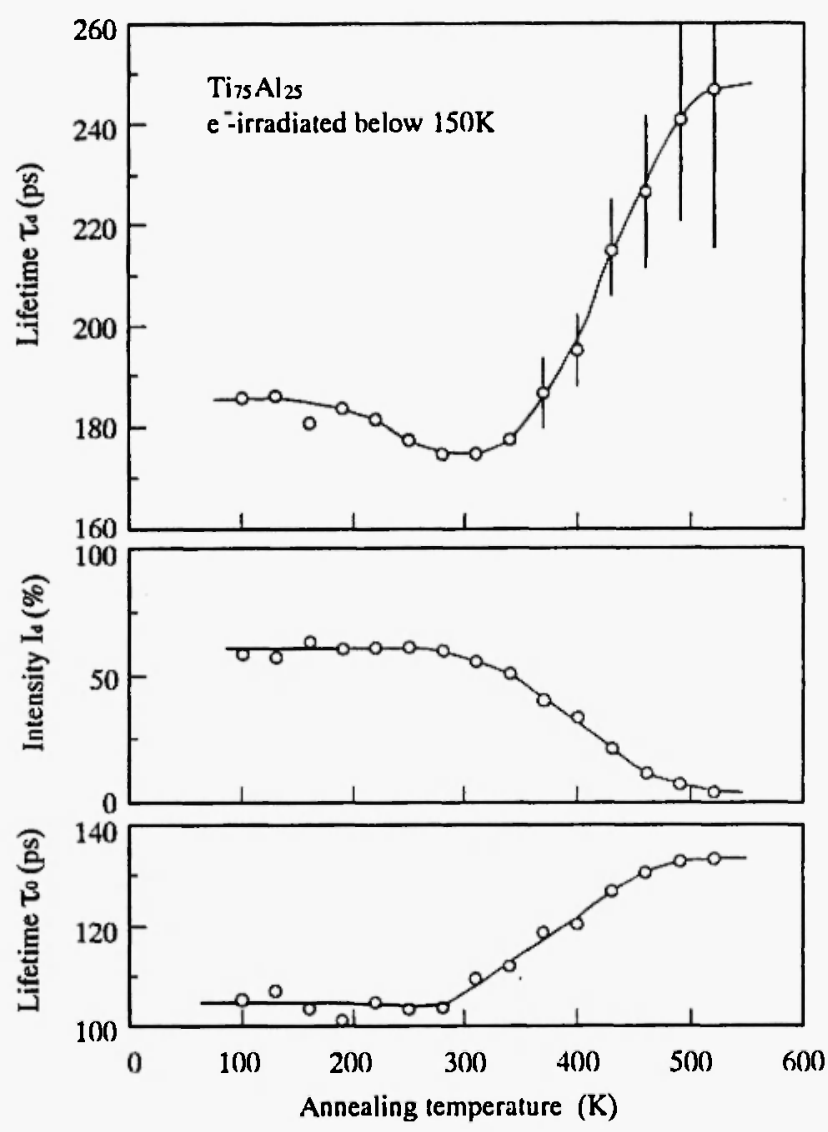

Fig.4: Changes in positron lifetime at defects $\left(\tau_{d}\right)$, the relative intensity of the defect component $\left(I_{\mathrm{d}}\right)$, and positron lifetime in the matrix $\left(\tau_{0}\right)$ on isochronal annealing (15min/30K) of $\mathrm{Ti}_{75} \mathrm{Al}_{25}$ irradiated below $150 \mathrm{~K}$ with $1 \mathrm{MeV}$ electrons to a total dose of $3.0 \times 10^{18} \mathrm{e}^{-\mathrm{cm}_{2}}$. 
positron lifetime at the secondary defects increases and exceeds $240 \mathrm{ps}$ in $\mathrm{Ti}_{3} \mathrm{Al}$, which is much higher than the corresponding lifetimes at vacancies.

It is interesting to note that no microvoids are formed by electron irradiation in the other two intermetallics in the Ti-Al system, $\mathrm{TiAl} / 32 /$ and $\mathrm{Al}_{3} \mathrm{Ti}$ 133/. Microvoids, i.e., uncollapsed vacancy clusters, are nucleated in the presence of impurity atoms that strongly interact with vacancies, and usually co-exist with intrinsic vacancy clusters $135 /$. The impurity that causes the formation of uncollapsed three-dimensional vacancy clusters in Ti3Al is most likely dissolved hydrogen atoms, whose concentration is of the order of $10^{3}$ at.ppm or more $/ 36 /$. It is well known that hydrogen causes the formation of microvoids in aluminum, where intrinsic vacancy clusters are collapsed faulted dislocation loops $/ 35 /$.

As has been shown above, it should be noticed that the positron annihilation method is very sensitive to vacancy and small vacancy clusters which are quite difficult to be studied by other techniques. There are some other works on positron annihilation which studied defect recovery processes in intermetallic compounds: $\mathrm{Ni}_{3} \mathrm{Al} / 37 /, \mathrm{Fe}_{3} \mathrm{Al} / 38 /, \mathrm{Ni}_{2} \mathrm{Si} / 39 /, \mathrm{NiSb}$ 139,40/.

\section{NEW METHOD FOR HIGH TEMPERATURE MEASUREMENTS}

In conventional positron lifetime measurements the $1.28 \mathrm{MeV} \gamma$-ray, which is emitted from a ${ }^{2} \mathrm{Na}$ positron source just after a positron emission, is used as the start signal for the time measurements. Consequently, the positron source should be placed at the same position of the specimen in which positrons annihilate, otherwise the time deference between a positron emission from the source and the positron implantation in the specimen blurs the positron lifetime spectrum. A positron source is usually prepared by evaporating a few microcuries of aqueous ${ }^{22} \mathrm{NaCl}$ onto a thin metal or plastic foil (typically $1 \mathrm{mg} \mathrm{cm}^{-2}$ ) and covering it with the same foil. The source is then sandwiched by two identical pieces of sample material to avoid the deterioration of lifetime spectrum mentioned above.

However, it is quite difficult or almost impossible to carry out lifetime measurements at high temperatures or under high pressures as long as we use the conventional sandwich configuration, because the positron source is usually sealed with epoxy or some other glue, which is not refractory enough. A sophisticated technique has been successfully adopted for high temperature measurements of some metals /41/: ${ }^{22} \mathrm{NaCl}$ is hermetically sealed by electron beam welding in the sample material under a vacuum. When the welding is tight, the sealed-source specimen can be heated close to the melting point of the material without any loss of radioactivity. The results shown in Fig. 5 were obtained by using the similar techniques. In spite of the achievement, the general difficulty of high temperature measurement still remains, since it is impossible to weld most intermetallics, semiconductors and ceramics.

In order to achieve in-situ studies of given matter at high temperatures, a new $\beta^{+}-\gamma$ coincidence lifetime spectrometer with the coincidence of energy-selected positron and the annihilation photon has been constructed by the author's group $/ 42,43 /$. The positron source is placed well apart from the sample which may be under extreme conditions, in order to prevent radioactive contamination (see Fig. 5). Consequently, the $1.28 \mathrm{MeV}$ photon from the positron source cannot be used as the start signal for the positron lifetime measurement. We therefore adopted the $\beta^{+}-\gamma$ coincidence technique /44/ which uses the positrons themselves for supplying start signals when passing a thin plastic scintillator.

To suppress the deterioration of time resolution due to the spread of TOF (time of flight) of positrons emitted from radioisotopes, we designed a beta-ray spectrometer to select positrons with a certain energy from a positron source. Two co-axial magnetic lenses are used for positron energy selection with higher efficiency. Only positrons within a certain energy-band can go through the hole in the baffle separating the two beta-ray spectrometers (see Fig. 5). Those positrons pass through the positron detector (a thin plastic scintillator connected with a light guide to a fast photomultiplier), and give a start signal to the coincidence circuit. The positrons that passed through the start detector are again selected in energy and trajectory, and focused onto the sample.

The whole apparatus is desk-top-sized. The total 
efficiency (more than $10^{-3}$ of positrons from the source can be focused on a specimen) is much superior to that expected in the case of re-acceleration of moderated positrons. The time resolution of the whole system with a $10 \mathrm{mCi}{ }^{22} \mathrm{Na}$ positron source is about $385 \mathrm{ps}$ (FWHM) as yet, which is worse than that of the conventional $\gamma-\gamma$ coincidence method (around 200ps FWHM typically) but bears comparison with former $\beta^{+}-\gamma$ coincidence

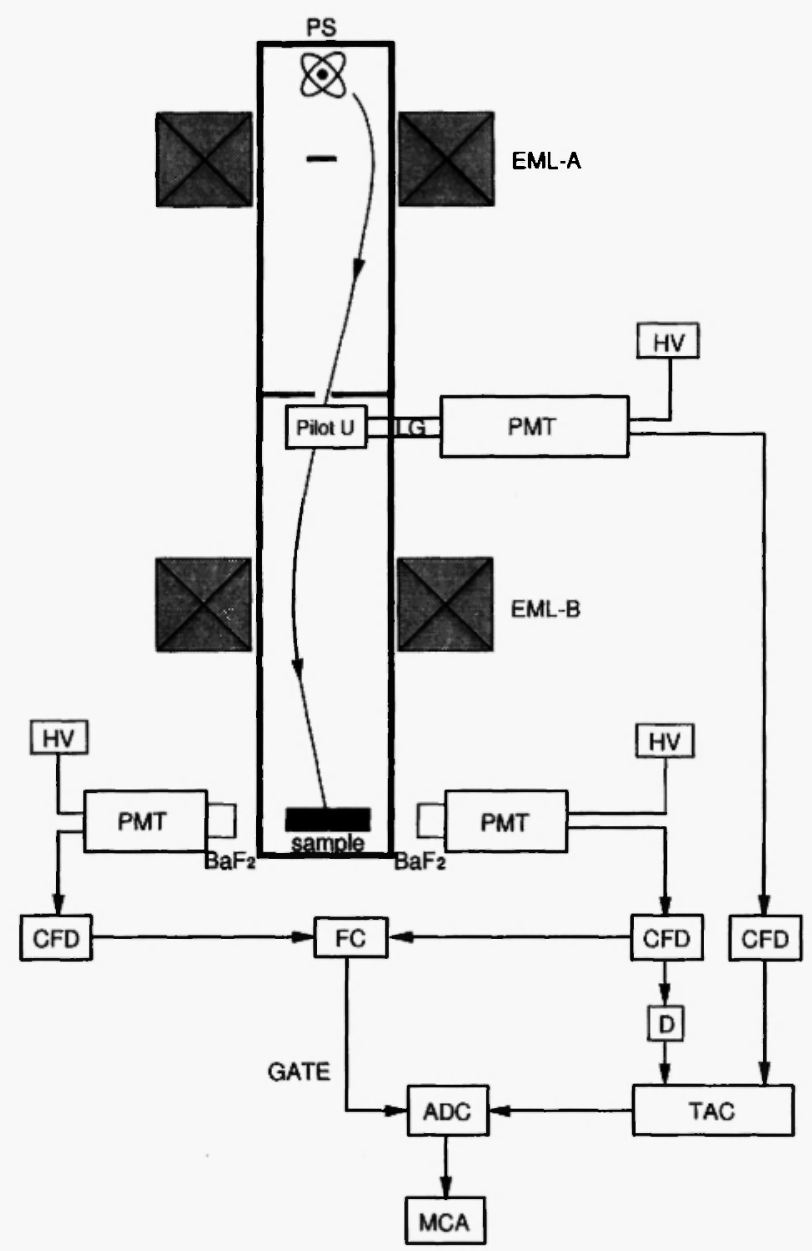

Fig.5: Schematic of the $\beta^{+}-\gamma$ coincidence lifetime spectrometer with the coincidence of energy-selected positron and the annihilation photon PS, Positron Source; EML, Electromagnetic lens; LG, Light Guide; PMT, Photo-Multiplier Tube; HV, High Voltage Power Supply; CFD, Constant Fraction discriminator; D, Nano-delay; TAC, Time Amplitude Converter; FC, Fast Coincidence; ADC, Analog-Digital Converter; MCA, Multi Channel Analyzer. lifetime spectrometers using close geometry between the positron source and a specimen $/ 42,45 /$.

\section{CONCLUDING REMARKS}

The distinctive characteristics of positron annihilation methods are:

1) The signals of defects are highly amplified

2) Defects with free volume are selectively detected

3) Species and sizes of atomic or nanometric scale defects are differentiated

4) The concentration of each defect is independently deduced

As has been shown in this article, these unique characteristics have already been applied in some high temperature materials. Hereafter, defects and their properties at high temperatures will be clarified in many important or potential refractory materials by using the new positron-lifetime spectrometer with fast-positron beam. Such information will be quite useful in the development of high temperature materials and processing.

\section{REFERENCES}

1. P. Hautojaervi (Ed.), Positrons in Solids, Springer-Verlag, Berlin, Heidelberg, New York, 1979.

2. W. Brandt and A. Dupasquier (Eds.), Positron Solid-State Physics, North-Holland Publishing Company, Amsterdam, New York, Oxford, 1983.

3. A. Dupasquier and A.P. Mills, Jr. (Eds.), Positron Spectroscopy of Solids, IOS Press, Amsterdam, Oxford, Tokyo, Washington DC, 1995.

4. L. Dorikens-Vanpraet, M. Dorikens and D. Segers (Eds.), Positron Annihilation, World Scientific, Singapore, 1989.

5. Zs. Kajcsos and Cs. Szeles (Eds.), Positron Annihilation, Materials Science Forum, 1992; 105-110.

6. Yuan-Jin He, Bi-Song Cao and Y.C. Jean (Eds.), Positron Annihilation, Materials Science Forum, 
$1995 ; 175-178$.

7. P.J. Schultz, G.R. Massoumi and P.J. Simpson (Eds.), Positron Beams for Solids and Surfaces, AIP Conference Proceedings, New York, 1990; 218.

8. M. Doyama, T. Akahane and M. Fujinami (Eds.), Proc. of $6^{\text {th }}$ Int. Workshop on Slow-Positron Beam Techniques for Solids and Surfaces, Appl. Surface Sci., 1995; 85.

9. W.B. Waeber, M. Shi and A.A. Manuel (Eds.), Proc. of $7^{\text {th }}$ Int. Workshop on Slow-Positron Beam Techniques for Solids and Surfaces, Appl. Surface Sci., 1997; 116.

10. W. Brandt, in: Positron Annihilation, A.T. Stewart and L.O. Roeling (Eds.), Academic Press, New York, 1967; 155.

11. B. Bergersen and M.J. Stott, Solid State Commun., 7, 1203 (1969).

12. D.C. Connors and R.N. West, Phys. Lett., A30, 24 (1969).

13. M. Doyoma, J. Phys. Soc. Japan, 33, 1495 (1972).

14. A. Seeger, Appl. Phys., 4, 183 (1974).

15. B.T.A. McKee, W. Trifshaeuser and A.T. Stewart, Phys. Rev. Lett., 28, 183 (1972).

16. R.O. Simmons and R.W. Balluffi, Phys. Rev., 117, 52 (1960).

17. H.E. Schaefer, in: Positron Annihilation, P.C. Jain, R.M. Singru and K.P. Gopinathan (Eds.), World Scientific, Singapore, 1985; 448.

18. K. Maier, in: Ref. 2, p. 265.

19. M. Yamaguchi and Y. Shirai, in: Physical Metallurgy and Processing of Intermetallic Compounds, N.S. Stoloff and V.K. Sikka (Eds.), Chapman and Hall, New York, 1995; 3.

20. Y. Shirai, Bulletin of the Japan Institute of Metals, 27, 869 (1988) [in Japanese].

21. Y. Shirai, F. Nakamura, $M$. Takeuchi, $K$. Watanabe and M. Yamaguchi, in Ref. 4, p. 488.

22. Y. Shirai, M. Takeuchi and M. Yamaguchi, unpublished.

23. H.E. Schaefer, R. Wuerschum, M. Sov, T. Zak, W.Z. Yu, W. Eckert and F. Banhart, Phys. Rev., 41, 11869 (1990).

24. H.E. Schaefer, R. Wuerschum and J. Bub, Materials Science Forum, 105-110, 439 (1992).

25. U. Brossmann, R. Wuerschum K. Badura and H.E.
Schaefer, Phys. Rev., 49, 6457 (1994).

26. F. Nakamura and J. Takamura, in: Point Defect and Defect Interactions in Metals, J. Takamura, M. Doyama and M. Kiritani (Eds.), University of Tokyo Press, Tokyo, 1982; 627.

27. Y.A. Chang and J.P. Neumann, Prog. Solid State Chem., 14, 221 (1982).

28. M. Shimotomai, T.M. Wang, T. Iwata and $M$. Doyama, in: Positron Annihilation, P.C. Jain, R.M. Singru and K.P. Gopinathan (Eds.), World Scientific, Singapore, 1985; 140.

29. Y. Shirai, I. Shishido and M Yamaguchi, unpublished.

30. Y. Ito, Y. Shirai, Y. Yamada and M. Yamaguchi, MRS Symposium Proceedings, Pittsburgh, 288, 275 (1993); Y. Shirai, Y. Ito and M. Yamaguchi, Materials Science Forum, 175-178, 529 (1995).

31. A.G. Balogh, L. Bottyan, G. Brauer, I. Dezsi and B. Molnar, J. Phys. Fi Met. Phys., 16, 1725 (1986).

32. Y. Shirai and M. Yamaguchi, Mat. Sci. Eng., A152, 173 (1992).

33. Y. Shirai, K. Masaki and $M$ Yamaguchi, Intermetallics, 2, 221 (1994).

34. Y. Shirai, T. Murakami, N. Ogawa and $M$. Yamaguchi, Intermetallics, 4, 31 (1996).

35. J. Takamura, Y. Shirai, K. Furukawa and F. Nakamura, Materials Science Forum, 15-18, 809 (1987).

36. D.S. Shih, G.K. Scarr and G.E. Wasielewski, Scripta Metallurgica, 23, 973 (1989).

37. T.M. Wang, $\mathbf{M}$ Shimotomai and $\mathbf{M}$ Doyama, $J$. Phys. F: Met. Phys., 14,37 (1984).

38. J.C. Wang, D.J. Liu, M.X. Chen and X.X. Cai, Scripta Met. Mat., 25, 2581 (1991).

39. A. Jennane, J. Bernardini, P. Moser and G. Moya, J. Alloys and Compounds, 188, 94 (1992).

40. A. Jennane, J. Bernardini, P. Moser and G. Moya, Appl. Phys., A59, 163 (1994).

41. K. Maier, M. Peo, B. Saile, H.E. Schaefer and A. Seeger, Phil. Mag., A40, 707 (1979); D. Herlach and K. Maier, Appl. Phys., 11, 197 (1976).

42. Y. Shirai, M. Sakamura, I. Shishido and $M$ Yamaguchi, J. Japan Inst. Metals, 59,679 (1995).

43. Y. Shirai, I. Shishido and M. Yuga, in preparation. 44. K. Maier and R. Myllylae, in: Proc. $5^{\text {th }}$ Int. Conf. 
Positron Annihilation, R.R. Hasiguti and K. Fujiwara (Eds.), The Japan Institute of Metals, Sendai 829 (1979).

45. H.E. Schaefer and W. Weiler, in Positron Annihilation, P.C. Jain, R.M. Singru and K.P. Gopinathan (Eds.), World Scientific, Singapore, $1985 ; 584$. 


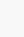

